\title{
Dual-Isotope Cryoimaging Quantitative Autoradiography: Investigating Antibody-Drug Conjugate Distribution and Payload Delivery Through Imaging
}

\author{
Ohad Ilovich*1, Mohammed Qutaish*1, Jacob Y. Hesterman ${ }^{1}$, Kelly Orcutt ${ }^{1}$, Jack Hoppin ${ }^{1}$, Ildiko Polyak ${ }^{1}$, Marc Seaman ${ }^{1}$, \\ Adnan O. Abu-Yousif ${ }^{2}$, Donna $\mathrm{Cvet}^{2}$, and Daniel P. Bradley ${ }^{2}$ \\ ${ }^{I}$ Invicro, LLC, Boston, Massachusetts; and ${ }^{2}$ Takeda Pharmaceuticals International Co., Cambridge, Massachusetts
}

See an invited perspective on this article on page 1459.

In vitro properties of antibody-drug conjugates (ADCs) such as binding, internalization, and cytotoxicity are often well characterized before in vivo studies. Interpretation of in vivo studies might be significantly enhanced by molecular imaging tools. We present here a dual-isotope cryoimaging quantitative autoradiography (CIQA) methodology combined with advanced 3-dimensional imaging and analysis allowing for the simultaneous study of both antibody and payload distribution in tissues of interest in a preclinical setting. Methods: TAK-264, an investigational ADC targeting anti-guanylyl cyclase $C$ (GCC), was synthesized using tritiated monomethyl auristatin $\mathrm{E}$. The tritiated ADC was then conjugated to diethylenetriaminepentaacetic acid, labeled with ${ }^{111} \mathrm{In}$, and evaluated in vivo in animals bearing GCC-positive and GCC-negative tumors. Results: CIQA revealed the time course of drug release from ADC and its distribution into various tumor regions that are less accessible to the antibody. For GCC-positive tumors, a representative section obtained $96 \mathrm{~h}$ after tracer injection showed only $0.8 \%$ of the voxels to have colocalized signal, versus over $15 \%$ of the voxels for a GCC-negative tumor section, suggesting successful and specific cleaving of the toxin in the GCC-positive lesions. Conclusion: The combination of a veteran established autoradiography technology with advanced image analysis methodologies affords an experimental tool that can support detailed characterization of ADC tumor penetration and pharmacokinetics.

Key Words: autoradiography; antibody-drug conjugate; dual isotope

J Nucl Med 2018; 59:1461-1466

DOI: 10.2967/jnumed.118.207753

\section{A}

ntibody-drug conjugates (ADCs) are targeted pharmaceuticals that comprise a tumor-targeting monoclonal antibody conjugated to a highly potent cytotoxic drug. This approach aims to assess the safety profile of highly potent drugs with limited tolerability $(1,2)$. Conjugation to monoclonal antibodies exploits the specific binding of the antibody as a selective delivery mechanism. This

Received Jan. 3, 2018; revision accepted Apr. 19, 2018.

For correspondence or reprints contact: Kelly Orcutt, Invicro, 27 Drydock Ave., Boston, MA 02210.

E-mail: orcutt@invicro.com

${ }^{*}$ Contributed equally to this work.

Published online May 4, 2018.

COPYRIGHT (c) 2018 by the Society of Nuclear Medicine and Molecular Imaging. process relies on a series of events including target binding, receptor-mediated cellular internalization, lysosomal processing of the ADC to release active drug, and drug action on cellular machinery (e.g., DNA or microtubule) (3-5). In addition to exerting cytotoxic effects on GCC-positive cells, the processed cytotoxic drug may also diffuse out and damage adjacent tumor cells, resulting in a bystander effect.

The ADC approach causes the payload (active drug) to take on the pharmacokinetic properties of the antibody. As such, ADCs encounter one of the major challenges of antibodies, heterogeneous distribution (6). As ADC technologies have advanced, the ability of a payload to exert bystander effects has been an area of active research. Linker designs aimed to release metabolites that are potent and cell-permeable have been developed with the goal of enabling cytotoxicity in proximal cells and greater efficacy in tumors with heterogeneous antigen expression or heterogeneous penetration of the ADC.

Traditionally, mechanism and efficacy are characterized in vitro for most new ADCs (7-9), with subsequent in vivo characterizations focused on pharmacokinetic and pharmacodynamic profiling, efficacy studies, and gross and destructive measurements of payload concentrations using mass spectrometry ex vivo. These techniques, although informative, do not allow for quantitative visualization of the extent of tumor penetration achieved by the antibody and processed drug.

The use of in vivo molecular imaging modalities such as PET combined with long-lived isotopes such as ${ }^{89} \mathrm{Zr}$ has allowed researchers to noninvasively evaluate parameters such as specific and nonspecific tumor uptake, target engagement, and offtarget accumulation in both preclinical and clinical settings (10). PET imaging, however, is limited to a spatial resolution on the order of millimeters, precluding evaluation of intratumoral distribution of antibody and drug. Matrix-assisted laser desorption/ ionization mass spectrometry is an emerging methodology for imaging the 3-dimensional (3D) distribution of drugs, peptides, and proteins ex vivo. Despite the high resolution and versatility of the method, it is hampered by the need for arduous optimization of sample preparation and acquisition for different molecules and tissues $(11,12)$ and by its lower sensitivity toward larger proteins. Autoradiography is a longstanding method for evaluating the distribution of target proteins using radiolabeled ligands ex vivo. The main uses of autoradiography have been the study of changes in neurotransmitter receptors in brain tissue (13-15) and the study of absorption, distribution, metabolism, and excretion $(16,17)$.

Cryoimaging quantitative autoradiography (CIQA) is an imaging methodology that involves the autoradiographic analysis of sections throughout a tissue of interest to determine the intratissue 
distribution of a radiolabeled molecule of interest. The radiolabeling of molecules with isotopes of significantly different halflives enables dual-isotope imaging, assisting in understanding how the molecules interact with or affect each other.

This paper describes the development of CIQA combined with 3D modeling to evaluate the intratumoral distribution of both antibody and drug by using a dual-labeled ADC ( ${ }^{111}$ In-diethylenetriaminepentaacetic acid [DTPA]- $\left.{ }^{3} \mathrm{H}-\mathrm{TAK}-264\right)$. This technology opens a new door to understanding how an ADC's dosing and linker structure together with tumor vasculature and antigen expression affect drug distribution and efficacy.

\section{MATERIALS AND METHODS}

\section{Radiochemistry}

${ }^{3} \mathrm{H}-\mathrm{MMAE}$ (185 MBq, $1 \mathrm{TBq} / \mathrm{mmol}$; Moravek Biochemicals) was conjugated to the fully human anti-GCC monoclonal antibody, 5F9, via a protease-cleavable linker (MMAE and linker technology licensed from Seattle Genetics). The conjugate 3H-TAK-264 was purified and formulated in $10 \mathrm{mM}$ histidine, $7.5 \%$ sucrose, $0.08 \%$ PS20, $\mathrm{pH} 5.2$, to obtain $70.3 \mathrm{MBq}$ of radiopharmaceutical with a radiochemical purity of $98.9 \%$, specific activity of $3.9 \mathrm{MBq} / \mathrm{mg}$, and a drug-toantibody ratio of $4.3: 1$.

${ }^{3} \mathrm{H}-\mathrm{TAK}-264$ (17.6 MBq/4.5 mg) was buffer-exchanged into HEPES buffer (0.1 M, pH 8.5) using an ultrafiltration tube (Amicon) and concentrated to approximately $5 \mathrm{mg} / \mathrm{mL}$. Diethylenetriaminepentaacetic acid (DTPA) dianhydride in dimethyl sulfoxide $(2 \mathrm{mg} / \mathrm{mL})$ was added to the protein solution to obtain a DTPA-to-antibody molar ratio of 20:1 and the reaction incubated at $4^{\circ} \mathrm{C}$ overnight. The reaction was buffer-exchanged into ammonium acetate buffer $(0.1 \mathrm{M}, \mathrm{pH} 6)$ and concentrated to approximately $5 \mathrm{mg} / \mathrm{mL}$.

A solution of ${ }^{111} \mathrm{InCl}_{3}(8 \mu \mathrm{L}, 300 \mathrm{MBq})$ was mixed with an equal volume of sodium acetate buffer $\left(0.1 \mathrm{M}, \mathrm{pH}\right.$ 6). DTPA- ${ }^{3} \mathrm{H}-\mathrm{TAK}-264$ $(1.89 \mathrm{mg}, 4.5 \mathrm{mg} / \mathrm{mL})$ was added to the reaction mixture and the reaction diluted to $1 \mathrm{~mL}$ with additional ammonium acetate buffer $(0.1 \mathrm{M}, \mathrm{pH}$ 6). The reaction mixture was left at room temperature for $30 \mathrm{~min}$, was stopped by addition of a $10 \%(\mathrm{v} / \mathrm{v})$ solution of ammonium acetate buffer $(0.1 \mathrm{M}, \mathrm{pH}$ 6) containing $50 \mathrm{mM}$ ethylenediaminetetraacetic acid, and was incubated for a further $5 \mathrm{~min}$ at room temperature. The labeling solution was formulated by dilution with saline to a final volume of $2 \mathrm{~mL}$. The final formulation was found have a radiochemical purity of $96.5 \%$ and specific activities of $296 \mathrm{MBq}$ $\left({ }^{111} \mathrm{In}\right)$ and $7.4 \mathrm{MBq}\left({ }^{3} \mathrm{H}\right)$ per $1.89 \mathrm{mg}$ of TAK-264 in $2 \mathrm{~mL}$.

${ }^{111}$ In-DTPA-5F9 and ${ }^{111}$ In-DTPA-TAK-264 were prepared from 5F9 and TAK-264 using similar strategies for DTPA conjugation and ${ }^{111}$ In labeling as described for the dual-labeled TAK-264. ${ }^{111} \mathrm{In}-$ DTPA-5F9 and ${ }^{111}$ In-DTPA-TAK-264 were obtained in radiochemical purities of $98 \%$ and $93 \%$ and specific activities of 2.46 and 2.51 $\mathrm{MBq} / \mu \mathrm{g}$, respectively.

The affinities of the DTPA-conjugated antibodies to GCC was compared with that of the parent antibodies using enzyme-linked immunosorbent assay and was found to be similar (data not shown).

\section{Animal Model}

All animal studies were performed at the University of Massachusetts and at Invicro with the approval of their respective Institutional Animal Care and Use Committees. The animals were kept at a temperature of $18^{\circ} \mathrm{C}-26^{\circ} \mathrm{C}$ and a relative humidity of $50 \% \pm 20 \%$, with intermittent light and dark cycles of $12 \mathrm{~h}$ and with food and water available ad libitum. Female SCID mice, 5-8 wk old, were implanted with HEK-293 GCC2 or HEK-293 cells $\left(5 \times 10^{6}\right.$ cells in $100 \%$ Matrigel [Corning]) subcutaneously. Tumor xenografts were allowed to grow to $200-1,500 \mathrm{~mm}^{3}$ for imaging studies and 180 $520 \mathrm{~mm}^{3}$ for CIQA studies.

\section{SPECT/CT Imaging}

The animals were imaged on a NanoSPECT/CT system (Bioscan, Inc.) using a 9-pinhole aperture (1.4-mm diameter) at 2 and $24 \mathrm{~h}$ after injection (20-min scans, each with 50 s/projection), at 48 and $96 \mathrm{~h}$ after injection (24-min scans, each with 60 s/projection), and at $144 \mathrm{~h}$ after injection (a 24- to 32-min scan with 60-80 s/projection). Scanning was performed with dual energy windows (154-188 and 221-270 $\mathrm{keV})$. Each SPECT scan was followed by a CT scan for anatomic reference ( 180 projections per rotation and $0.5 \mathrm{~s} /$ projection). Anesthesia during imaging was induced with $4 \%-5 \%$ isoflurane and maintained with $1 \%-2 \%$ isoflurane $(0.5-1 \mathrm{~L} / \mathrm{min})$.

Animals bearing HEK-293 GCC2 tumors $(n=8)$ were anesthetized, injected with either ${ }^{111}$ In-DTPA-5F9 $(19.8 \pm 0.55 \mathrm{MBq}, n=4)$ or ${ }^{111}$ In-TAK-264 (19.4 $\left.\pm 0.85 \mathrm{MBq}, n=4\right)$, and imaged at the time points described above to evaluate biodistribution profiles.

\section{Biodistribution and Autoradiography}

Tumor-bearing animals (HEK-293 $[n=8]$ and HEK-293 GCC2 $[n=8])$ were anesthetized and injected with $15.5 \pm 0.6$ and $14.1 \pm$ $1.4 \mathrm{MBq}$, respectively, of ${ }^{111}$ In-DTPA- ${ }^{3} \mathrm{H}-\mathrm{TAK}-264$. Two animals from each group were euthanized at each time point $(1,8,24$ and $96 \mathrm{~h}$ after injection), and their tumors were resected. On resection, the tumors were immediately blocked in 5\% carboxymethyl-cellulose on crushed dry ice. ${ }^{14} \mathrm{C}$-2-doxyglucose-spiked India ink fiducial markers were inserted into the blocks, which were then stored in a $-20^{\circ} \mathrm{C}$ freezer. High-resolution optical images of each $30-\mu \mathrm{m}$ section were acquired using a Canon EOS 70D camera, and each tenth section was stored on 3M tape (Scotch Label Protection Tape 821), dehydrated, and exposed to phosphor image screens (BAS-SR; GE Healthcare) for 2-3 h, which were scanned using a BAS5000 image acquisition system to determine ${ }^{111}$ In content.

${ }^{3} \mathrm{H}$ signal was overshadowed by ${ }^{111}$ In signal and by the signal of the long-lived radioactive contaminants in the ${ }^{111} \mathrm{InCl}_{3}$ used for labeling. ${ }^{111}$ In signal was removed by exploiting the fact that the physical decay of ${ }^{111} \mathrm{In}$ is faster than that of ${ }^{3} \mathrm{H}$. In this study, ${ }^{3} \mathrm{H}$ signal was acquired after a delay of more than $10{ }^{111}$ In half-lives. Due to the presence of long-lived contaminants following ${ }^{111} \mathrm{In}$ decay, the ${ }^{3} \mathrm{H}$ signal was extrapolated from the total signal as described in the "Image Analysis" section.

\section{Image Analysis}

SPECT/CT. SPECT images were reconstructed using a scanner manufacturer-provided multipinhole ordered-subsets expectation maximization algorithm. Regions of interest were delineated and quantified by manual fitting of constant volumes of interest to the heart $\left(243 \mathrm{~mm}^{3}\right)$, liver $\left(143 \mathrm{~mm}^{3}\right)$, spleen $\left(75 \mathrm{~mm}^{3}\right)$, left and right kidneys $\left(500 \mathrm{~mm}^{3}\right)$, and muscle $\left(50 \mathrm{~mm}^{3}\right)$ using VivoQuant (Invicro). Tumor regions of interest $\left(130-1,600 \mathrm{~mm}^{3}\right)$ were drawn manually and maximized to the size of the lesion. Nontumor volumes of interest were fitted on the basis of the CT data and centered on the peakactivity profile.

Autoradiography. Autoradiography image analysis was performed using VivoQuant. Individual white-light images were aligned into a 3D dataset using a fiducial marker-based registration approach. First, the ${ }^{14} \mathrm{C}$-ink fiducial centers within each slice were detected by applying a normalized cross correlation with a disk image. Then, a rigid-body transformation was estimated to align these images to a reference image. Least-squares distance error was used as the optimization parameter for estimating the registration parameters. After registration, variation in intensity throughout the image stack was corrected by a histogram-based color-matching algorithm (18) to complete the final 3D white-light dataset.

${ }^{111} \mathrm{In}$ and ${ }^{3} \mathrm{H}$ autoradiography images were intensity-calibrated using a set of standards included in each plate. Linear regression yielded 


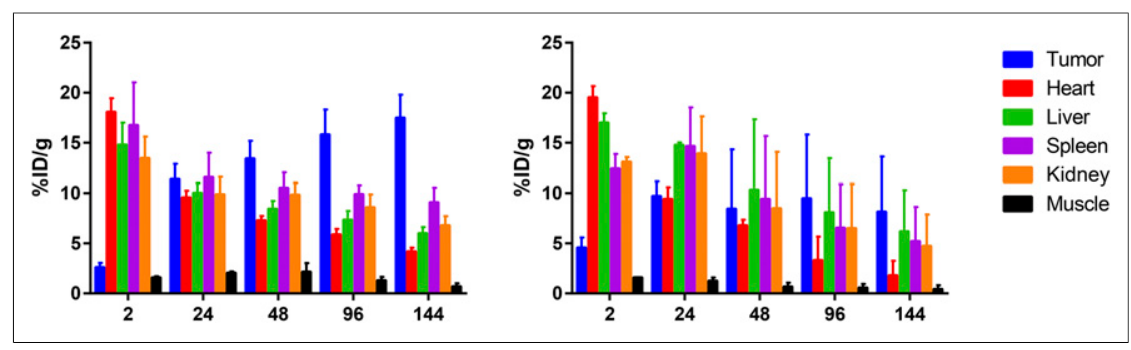

FIGURE 1. Distribution of ${ }^{111}$ In-DTPA-5F9 (left) and ${ }^{111}$ In-DTPA-TAK-264 (right) in mice bearing HEK-293 GCC2 subcutaneous tumors. Data are mean \pm SD ( $n=3-4$ for all time points).

a plate-specific calibration parameter to convert intensity values into units of $\mathrm{kBq} / \mathrm{g}$. Reconstruction of ${ }^{111}$ In tumor $3 \mathrm{D}$ volumes started by splitting the calibrated plates containing multiple tumor section into individual ones. Next, the ${ }^{14} \mathrm{C}$-ink fiducial markers were detected using the same algorithm as used for the white-light images. The fiducial markers were used to coregister the plates into a 2-dimensional (2D) stack. Because autoradiography images were captured every 10 white-light slices, linear interpolation was performed to produce the final 3D autoradiography dataset with isotropic voxel size. Fiducial centers from the white-light stack and the ${ }^{111}$ In stack were used to register the white-light volume to its corresponding ${ }^{111} \mathrm{In}$ volume using a Procrustes transformation that allows translation, rotation, and scaling. Both volumes were resampled to $0.025 \times 0.025 \times$ $0.025 \mathrm{~mm}$ isotropic voxels.

The original strategy for dual-isotope imaging was to quantify the higher-energy and shorter-lived ${ }^{111}$ In signal, allowing for sufficient ${ }^{111}$ In decay (30 d, 11 half-lives), followed by quantification of the ${ }^{3} \mathrm{H}$ signal. The presence of small quantities $(<0.1 \%)$ of long-lived, $\gamma$-emitting isotope contaminants $\left({ }^{114 \mathrm{~m}} \mathrm{In}\right.$ and ${ }^{65} \mathrm{Zn}$ with half-lives of 50 and $244 \mathrm{~d}$, respectively) required an alternate quantification strategy. After allowing 2 mo for ${ }^{111}$ In decay to the background level, the tissues were quantified twice for radioactivity signal. First, the total radioactive content ( $\gamma$-emitting contaminants plus tritium) was quantified by exposing tissues to standard phosphor imaging screens. Exposures were repeated with the screens covered in foil to block the tritium signal, effectively quantifying contaminant signal only. The relative intensity of the ${ }^{111} \mathrm{In}$ standards (containing only contaminants; no measurable ${ }^{111} \mathrm{In}$ signal and no ${ }^{3} \mathrm{H}$ signal) in images on phosphor imaging screens with and without aluminum foil was used to estimate the contribution of the ${ }^{111} \mathrm{In}$ contaminants to ${ }^{3} \mathrm{H}$ signal in tumors. Images of the standards acquired with and without foil were corrected for differences in exposure time, and a correction factor based on the relative intensity of the standards was calculated. This correction factor was used to scale the foil-covered (contaminant) image. After the 2 plates had been registered, a smoothing step (2D gaussian kernel with a full width at half maximum of $0.150 \mathrm{~mm}$ ) was applied to the scaled, foilcovered image to preserve structure in the contaminant image while reducing the impact of noise. The scaled, smoothed contaminant image was subtracted from the image without foil to remove the signal contribution from the contaminants and yield the tritium tissue signal. To improve voxel-level comparison between the ${ }^{3} \mathrm{H}$ and ${ }^{111}$ In autoradioluminograms, a second smoothing step (3D gaussian kernel with a full width half maximum of $0.100 \mathrm{~mm}$ ) was applied to the ${ }^{3} \mathrm{H}$ images.

\section{RESULTS}

\section{Evaluation of Intratumoral Accumulation of ${ }^{111}$ In-DTPA-5F9 and ${ }^{111}$ In-DTPA-TAK-264 Using SPECT/CT}

On SPECT/CT images, ${ }^{111}$ In-DTPA-5F9 showed a continuous accumulation in tumors, averaging 16-18 \% ID/g at $144 \mathrm{~h}$ (Supplemental Figs. 1 and 2; supplemental materials are available at http://jnm. snmjournals.org). ${ }^{111} \mathrm{In}$-TAK-264 peak tumor uptake was reached at $96 \mathrm{~h}$ after injection (11-13\% $\mathrm{ID} / \mathrm{g})$ and remained constant at $144 \mathrm{~h}$ after injection (Fig. 1). The differences in tumor uptake between ${ }^{111}$ In-DTPA-5F9 and ${ }^{111}$ In-TAK-264 were statistically significant at the first and last time points $(2 \mathrm{~h}$ and $144 \mathrm{~h}$, with $P=0.015$ and $P=0.007$, respectively). Significant differences in plasma clearance (calculation based on the heart region of interest, which approximates blood pool radioactivity) were apparent $(P=0.03)$.

On the basis of these data, the CIQA experiment was conducted on tumors resected at $1,8,24$ and $96 \mathrm{~h}$ after injection using the HEK-293 GCC2 tumor model with the HEK-293 tumor as the GCC-negative control.

\section{Evaluation of Intratumoral Distribution of 5F9 and Its MMAE Payload Using CIQA Plus 3D Image Reconstruction}

Figure 2 presents representative autoradiography sections. Maximum-intensity projections were used to visualize tumors in 3 dimensions. Supplemental Fig. 3 is a diagram of the acquisition and reconstruction process, and Supplemental Video 1 shows the 3D reconstructed maximum-intensity projections of GCC-positive and -negative tumors at $96 \mathrm{~h}$ after injection.

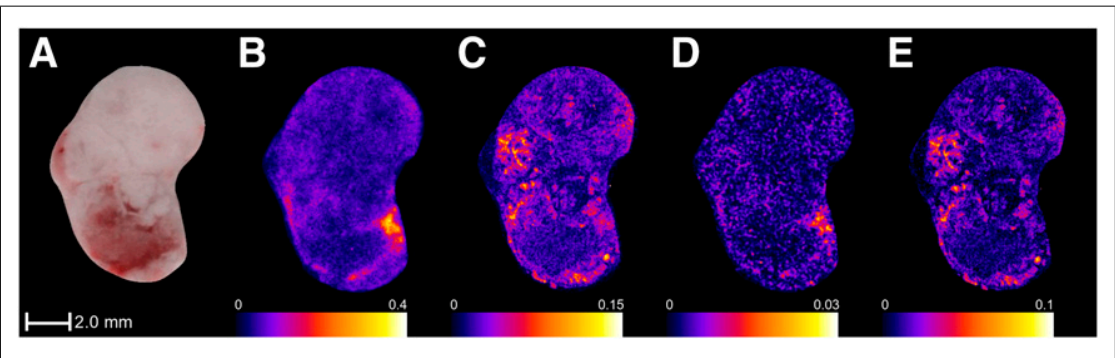

FIGURE 2. Representative HEK-293 GCC2 tumor sections showing subtraction technique used to compensate for presence of long-lived contaminants in ${ }^{111} \mathrm{In}$-labeled ADC. Voxels are $0.025 \times$ $0.025 \times 0.025 \mathrm{~mm}$. Images are displayed for qualitative review in units of photostimulated luminescence and include white-light image $(A)$ and images showing ${ }^{111}$ In signal $(B)$, total $\left({ }^{3} \mathrm{H}+\right.$ contaminants) signal (C), contaminant-only signal (D), and isolated ${ }^{3} \mathrm{H}$ signal $(\mathrm{E})$.

\section{Demonstration of Differential Distribution of MMAE in GCC-Negative and -Positive Tumors on CIQA}

Representative tumor sections at 1, 24, and $96 \mathrm{~h}$ after injection are shown in Figure 3. At 1 and $8 \mathrm{~h}$ (data not shown) after injection, ${ }^{3} \mathrm{H}$ and ${ }^{111} \mathrm{In}$ signals were very colocalized in both GCC-positive and GCC-negative tumors (Fig. 3A). At $24 \mathrm{~h}$, ${ }^{3} \mathrm{H}$ and ${ }^{111}$ In signals were still very colocalized in the GCC-negative tumors but were starting to diverge in the GCC-positive tumors (Fig. 3B). By $96 \mathrm{~h},{ }^{3} \mathrm{H}$ and ${ }^{111}$ In showed a visible drop in signal 


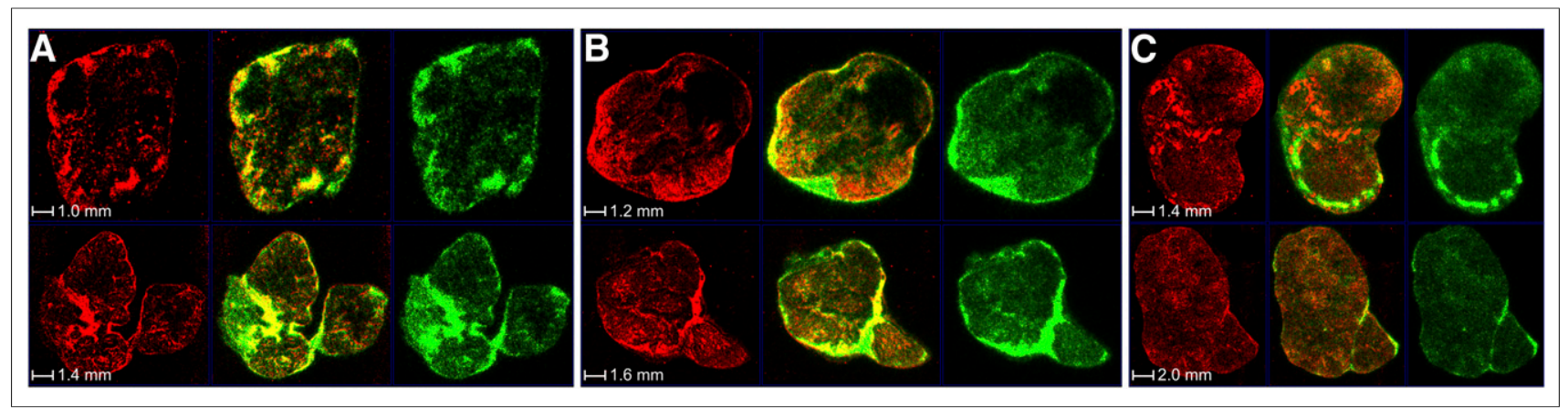

FIGURE 3. Representative sections of HEK-293 GCC2 tumors (top) and HEK-293 tumors (bottom) excised at $1 \mathrm{~h} \mathrm{(A),} 24 \mathrm{~h} \mathrm{(B)}$, and $96 \mathrm{~h}(\mathrm{C})$ after tracer injection. Voxels are $0.025 \times 0.025 \times 0.025 \mathrm{~mm}$. ${ }^{3} \mathrm{H}$ signal is red, ${ }^{111} \mathrm{In}$ signal is green, and both signals coregistered is yellow. Strikingly, image of HEK-293 GCC2 at 24 h shows initial diffusion of drug away from antibody accumulation site and deeper into tumor.

intensity in the GCC-negative tumors and exhibited some divergence (Fig. 3C). In the GCC-positive tumors, the ${ }^{3} \mathrm{H}$ and ${ }^{111} \mathrm{In}$ signals were more divergent at $96 \mathrm{~h}$ than at $24 \mathrm{~h}$, with the ${ }^{3} \mathrm{H}$ signal penetrating more deeply into the tumor (Fig. 3C). Both tumor types demonstrated significant heterogeneity in the distribution of the antibody signal $\left({ }^{111} \mathrm{In}\right)$, as expected for compounds of high molecular weight $(19,20)$.

The ${ }^{111}$ In signal in HEK-293 GCC2 tumors steadily increased over the 96-h period, whereas the ${ }^{111}$ In signal in HEK-293 tumors decreased as the nonspecifically bound tracer was slowly washed out of the tumor (Fig. 4). A similar trend was clearly visible in the MMAE-affiliated ${ }^{3} \mathrm{H}$ signal, with the main difference being that the tritium signal spread into the center of the HEK-293 GCC2 tumors whereas the ${ }^{111}$ In signal remained localized at the tumor edges.

\section{Demonstration of Bystander Effect via Colocalization Analysis of ${ }^{3} \mathrm{H}$ and ${ }^{111} \mathrm{In}$ in GCC-Positive Tumors}

Differences in background and photon scatter characteristics in autoradioluminograms of the 2 isotopes can affect assessment of colocalization of the ${ }^{111} \mathrm{In}$ and ${ }^{3} \mathrm{H}$ signals (21). Analysis of signal overlap focused on, first, differences in overall signal intensity between tumor lines and, second, the extent of colocalization of the ${ }^{111} \mathrm{In}$ and ${ }^{3} \mathrm{H}$ signals in the highest-intensity voxels for each tumor line. The fraction of the highest-intensity voxels that are common to both isotopes provides a measure of colocalization. Figure 5 shows a 2D scatterplot of the threshold-constrained voxels for tumors from each cell line at $96 \mathrm{~h}$. The thresholds in the HEK-293 GCC2 tumors were 2- to 3-fold higher than those in the HEK-293 tumors for both ${ }^{111} \mathrm{In}$ and ${ }^{3} \mathrm{H}$. Additionally, these high-intensity voxels were almost completely disparate between the 2 isotopes for the HEK-293 GCC2 tumors (0.8\% overlap) whereas a colocalization fraction of $15.5 \%$ was observed between the highestintensity voxels in the HEK-293 tumors (Supplemental Fig. 4 shows the same data without the thresholding). Taken together, these data demonstrate greater uptake of TAK-264 in HEK-293 GCC2 tumors than in GCC-negative HEK-293 tumors. In addition, the disparate signal observed between the 2 isotopes in the GCC-positive tumors is indicative of binding, internalization, metabolism, and release of MMAE.

\section{DISCUSSION}

Preliminary SPECT/CT imaging studies were performed on ${ }^{111} \mathrm{In}$-labeled parent antibody and ADC to examine the tissue distribution of the compounds over time. With the relevant distribution information at hand, the ADC was dual-labeled with both ${ }^{111}$ In (antibody) and ${ }^{3} \mathrm{H}$ (MMAE) and CIQA was performed in both HEK-293 GCC2 (GCC-positive) and HEK-293 (GCC-negative) tumor-bearing animals at different times after injection.

Analysis of the highest-intensity voxels allowed us to investigate the colocalization of the 2 signals. For GCC-positive tumors, a representative section obtained at $96 \mathrm{~h}$ after 


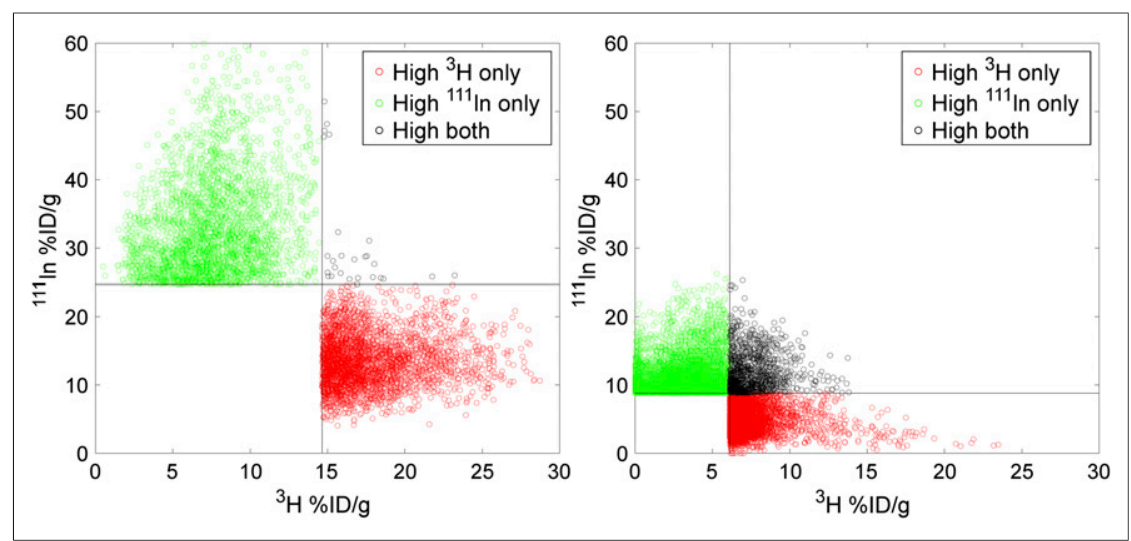

FIGURE 5. Colocalization analysis of top $3 \%$ of highest-intensity voxels for GCC-positive tumors (left) and GCC-negative tumors (right). Voxels with high ${ }^{3} \mathrm{H}$ signal (MMAE), high ${ }^{111}$ In signal (antibody), or both are shown.

tracer injection showed that only $0.8 \%$ of the voxels had colocalized signal, versus over $15 \%$ of the voxels for a GCC-negative tumor section. These results are in line with our expectation that GCCpositive tumor cells would internalize and metabolize the ADC, allowing the free drug to diffuse across the tumor and affect untargeted cells in what is known as the bystander effect (22). In GCC-negative tumors, there is no target-mediated internalization; thus, a high percentage of colocalized signal is expected. Macropinocytosis has been shown to occur in response to growth factor stimulation and constitutively in some cell types and may cause the decoupling of ${ }^{111} \mathrm{In}$ and ${ }^{3} \mathrm{H}$ signals observed in the GCC-negative tumors.

The indium contaminants made the CIQA process more difficult, time-consuming, and expensive than desired. A good companion isotope for ${ }^{3} \mathrm{H} /{ }^{14} \mathrm{C}$ would have a half-life of several days (matching well the biologic half-lives of antibodies yet enabling a reasonable decay period). The isotope of choice should residualize in tumors to minimize the redistribution of metabolites, emit lowenergy $\gamma$-energies to retain autoradiography resolution, have a high specific activity, and have short-lived contaminants. ${ }^{67} \mathrm{Ga}$ is a residualizing isotope with established radiochemistry (23-25), a half-life of $3.3 \mathrm{~d}$, specific activity of $148 \mathrm{TBq} / \mathrm{mmol}$, low-energy $\gamma$-emissions, and a minimal presence $(<0.6 \%)$ of short-lived contaminants $\left({ }^{66} \mathrm{Ga}\right.$ and ${ }^{68} \mathrm{Ga}$, with half-lives of $9.5 \mathrm{~h}$ and $68 \mathrm{~min}$, respectively). The use of ${ }^{67} \mathrm{Ga}$ as a surrogate for ${ }^{111} \mathrm{In}$ has been validated internally to ensure that future CIQA studies will be more streamlined and cost-effective.

To avoid potential translocation and loss of the MMAE due to the tissue handling, the animals in this study were not perfused before tumor harvesting (26). In the interpretation of the results, it is important to consider that the tumor signal includes contributions from both tumor tissue and blood pool.

Despite some limitations such as the use of radioactive compounds, an inability to distinguish between the different radioactive metabolites, and the need to allow extended periods for radioisotope decay, CIQA is well positioned when compared with imaging methodologies of similar scope. Fluorescence imaging is an inexpensive and straightforward methodology that allows for depth-limited in vivo imaging combined with superior ex vivo resolution. However, ADC conjugation to fluorescent dyes affects pharmacokinetics significantly, even in low conjugate-to-antibody ratios (27), and the dyes themselves may undergo quenching after cellular uptake, making analysis challenging. Mass spectrometry imaging is an attractive modality but has a low sensitivity for detecting large proteins over a tissue background, and because different metabolites will have different masses and may be conjugated to DNA or proteins, the signal may become more diluted over time and specific standards would be required for the expected metabolites. A final possibility is immunohistochemical identification of both drug and antibody, but this methodology requires specialized antibody pairs that may not have affinity to the formed metabolites and may produce signals that are more difficult to quantify.

\section{CONCLUSION}

CIQA is a unique and powerful method to evaluate the distribution and efficacy of ADCs in vivo. CIQA allows the evaluation of ADC metabolism in tumors of varying antigen expression and organs (e.g., liver) known to have off-target toxicities. The results of this study are a proof of concept demonstrating that the CIQA technology can help explain the spatiotemporal dynamics of different antibodies, linkers, and drug conjugates.

\section{DISCLOSURE}

Ohad Ilovich, Mohammed Qutaish, Jacob Hesterman, Kelly Orcutt, Jack Hoppin, Ildiko Polyak, and Marc Seaman were employed by Invicro LLC at the time the experiments were conducted. Adnan Abu-Yousif, Donna Cvet, and Daniel Bradley were employed by Takeda Pharmaceuticals International at the time the experiments were conducted. No other potential conflict of interest relevant to this article was reported.

\section{ACKNOWLEDGMENTS}

We are extremely grateful to Vijay Gottumukkala, Paige Czarnecki, Mihaela Plesescu, Ozlem Yardibi, Rick Coelho, Merryl Lobo, and Chris Graul for the execution and operationalizing of the study and the highly useful advice they shared.

\section{REFERENCES}

1. Blum RH, Wittenberg BK, Canellos GP, et al. A therapeutic trial of maytansine. Cancer Clin Trials. 1978;1:113-117.

2. Mirsalis JC, Schindler-Horvat J, Hill JR, Tomaszewski JE, Donohue SJ, Tyson CA. Toxicity of dolastatin 10 in mice, rats and dogs and its clinical relevance. Cancer Chemother Pharmacol. 1999;44:395-402.

3. Ducry L, Stump B. Antibody-drug conjugates: linking cytotoxic payloads to monoclonal antibodies. Bioconjug Chem. 2010;21:5-13.

4. Nolting B. Linker technologies for antibody-drug conjugates. Methods Mol Biol. 2013;1045:71-100.

5. Bareford LM, Swaan PW. Endocytic mechanisms for targeted drug delivery. Adv Drug Deliv Rev. 2007;59:748-758.

6. Thurber GM, Schmidt MM, Wittrup KD. Antibody tumor penetration: transport opposed by systemic and antigen-mediated clearance. Adv Drug Deliv Rev. 2008;60: 1421-1434.

7. Kularatne SA, Deshmukh V, Ma J, et al. A CXCR4-targeted site-specific antibody-drug conjugate. Angew Chem Int Ed Engl. 2014;53:11863-11867.

8. Tai Y-T, Mayes PA, Acharya C, et al. Novel anti-B-cell maturation antigen antibody-drug conjugate (GSK2857916) selectively induces killing of multiple myeloma. Blood. 2014;123:3128-3138.

9. Golfier S, Kopitz C, Kahnert A, et al. Anetumab ravtansine: a novel mesothelin-targeting antibody-drug conjugate cures tumors with heterogeneous 
target expression favored by bystander effect. Mol Cancer Ther. 2014;13:15371548.

10. Lamberts LE, Williams SP, Terwisscha van Scheltinga AGT, et al. Antibody positron emission tomography imaging in anticancer drug development. J Clin Oncol. 2015;33:1491-1504.

11. Schwamborn K, Caprioli RM. MALDI imaging mass spectrometry: painting molecular pictures. Mol Oncol. 2010;4:529-538.

12. Quiason CM, Shahidi-Latham SK. Imaging MALDI MS of dosed brain tissues utilizing an alternative analyte pre-extraction approach. J Am Soc Mass Spectrom. 2015;26:967-973.

13. Kupila J, Karkkainen O, Laukkanen V, et al. $\left[{ }^{3} \mathrm{H}\right]$ Ifenprodil binding in postmortem brains of Cloninger type 1 and 2 alcoholics: a whole-hemisphere autoradiography study. Psychiatry Res. 2015;231:197-201.

14. Yeh SH-H, Lin M-H, Kong F-L, et al. Evaluation of inhibitory effect of recreational drugs on dopaminergic terminal neuron by PET and whole-body autoradiography. Biomed Res Int. 2014;2014:157923.

15. Fang C-K, Chen H-W, Wang W-H, Liu R-S, Hwang J-J. Acute effects of three club drugs on the striatum of rats: evaluation by quantitative autoradiography with $\left[{ }^{18}\right.$ F]FDOPA. Appl Radiat Isot. 2013;77:153-159.

16. Solon EG. Autoradiography techniques and quantification of drug distribution. Cell Tissue Res. 2015;360:87-107.

17. Solon EG. Use of radioactive compounds and autoradiography to determine drug tissue distribution. Chem Res Toxicol. 2012;25:543-555.

18. Grundland M. Color histogram specification by histogram warping. Proc SPIE. 2005;5667:610-621.
19. Orcutt KD, Adams GP, Wu AM, et al. Molecular simulation of receptor occupancy and tumor penetration of an antibody and smaller scaffolds: application to molecular imaging. Mol Imaging Biol. 2017;19:656-664.

20. Wilks MQ, Knowles SM, Wu AM, Huang S-C. Improved modeling of in vivo kinetics of slowly diffusing radiotracers for tumor imaging. J Nucl Med. 2014;55:1539-1544.

21. Johnston RF, Pickett SC, Barker DL. Autoradiography using storage phosphor technology. Electrophoresis. 1990;11:355-360.

22. Li F, Emmerton KK, Jonas M, et al. Intracellular released payload influences potency and bystander-killing effects of antibody-drug conjugates in preclinical models. Cancer Res. 2016;76:2710-2719.

23. Morais M, Cantante C, Gano L, et al. Biodistribution of a ${ }^{67} \mathrm{Ga}$-labeled anti-TNF VHH single-domain antibody containing a bacterial albumin-binding domain (Zag). Nucl Med Biol. 2014;41(suppl):e44-e48.

24. Alirezapour B, Jalilian A, Bolourinovin F, Moradkhani S. Production and quality control of $\left[{ }^{67} \mathrm{Ga}\right]$-DOTA-trastuzumab for radioimmunoscintigraphy. Iran J Pharm Res. 2013;12:355-366.

25. Eder M, Knackmuss S, Le Gall F, et al. ${ }^{68}$ Ga-labelled recombinant antibody variants for immuno-PET imaging of solid tumours. Eur J Nucl Med Mol Imaging. 2010;37:1397-1407.

26. Walter SE. Drug Localization in Tissues and Cells. Chapel Hill, NC: IDDC Press; 2003:26-27.

27. Cilliers C, Nessler I, Christodolu N, Thurber GM. Tracking antibody distribution with near-infrared fluorescent dyes: impact of dye structure and degree of labeling on plasma clearance. Mol Pharm. 2017;14:1623-1633. 